\title{
Interface Placement in Constructing Widest Spanning Tree for Multi-Channel Multi-Interface Wireless Mesh Networks
}

\author{
Hon Sun Chiu, Kwan L. Yeung and King-Shan Lui \\ Department of Electrical and Electronic Engineering \\ The University of Hong Kong, Hong Kong, PRC. \\ Tel: (852) 2857-8493 Fax: (852) 2559-8738 \\ Email: \{hschiu,kyeung,kslui\}@eee.hku.hk
}

\begin{abstract}
Widest spanning tree is a broadcast tree with its bottleneck link bandwidth maximized. It provides a cost effective broadcasting solution in multi-channel multi-interface wireless mesh networks. To find the widest spanning tree, existing algorithms jointly consider channel assignment, routing and scheduling while assuming the number of network interface cards (NICs) at each node is given. In this paper, we treat the number of NICs at each node as a design parameter, whereas the total number of NICs in the system is given. By properly placing more NICs to more "critical" nodes, the bandwidth of the spanning tree can be further increased. To this end, a new Integer Linear Programming (ILP) is formulated for solving the widest spanning tree problem based on joint optimization of interface placement, channel assignment, routing and scheduling. Numerical results show that interface placement provides a significant boost to the bandwidth of the widest spanning tree found.
\end{abstract}

Keywords - multiple channels, multiple interfaces, interface placement, wireless mesh network, widest spanning tree.

\section{INTRODUCTION}

Due to the inexpensive and easy to deploy characteristics, wireless mesh networks (WMNs) has become a promising technology for extending the coverage of the fixed broadband Internet. A WMN consists of stationary wireless mesh routers, which are connected to one another in a multi-hop manner to form a wireless backbone. With the increasing popularity of WMN, many applications designed for the Internet have been adopted by WMN [1], which quickly throttles the capacity of the network. While minimizing energy consumption, or equivalently maximizing the network lifetime, is critical for wireless ad hoc networks [2-7] where nodes are powered by battery, energy is generally not a limitation for WMNs. In contrast, current research efforts in WMNs focus at efficient resources utilization for improving the network capacity to meet the ever increasing traffic demand [9-19].

In a wireless network, nodes are not directly connected by wires, which make the transmission behavior different from the wired network. Transmissions in wireless network are delivered through the shared wireless broadcast medium, where all neighbors can receive the same copy of data with the source node only transmitting once. This is known as wireless broadcast advantage (WBA) [7]. On the other hand, wireless transmission also induces interference. A node has to defer its transmission if there is another on-going transmission within its interference range. Mutual interference severely limits the capacity of a wireless network. To improve the network capacity, transmissions within interference range can be assigned to use different orthogonal channels. The network capacity can be further enhanced by equipping each node with multiple network interface cards (NICs) such that more parallel transmissions on different channels can occur [8].

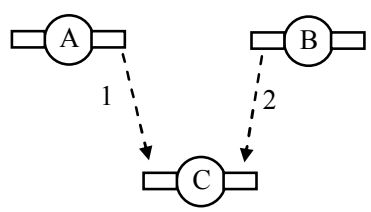

(a) Fixed interfaces/node

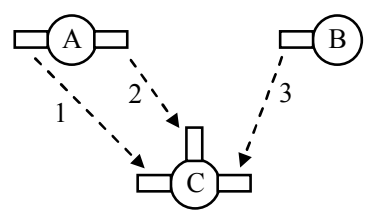

(b) Interface Placement
Fig. 1. Two scenarios of possible interface placement with 6 interfaces

Broadcasting is fundamental to important applications such as exchanging routing information, content update for a distributed servers system, cache server update for better web surfing performance, and more recently distributing IP-TV. Many spanning tree protocols have been proposed for multi-channel multi-interface WMNs. The work in $[9,10]$ aims at finding a spanning tree for Internet access, where the Internet gateway node is also the root of the tee. Channels are assigned to the wireless nodes such that interference is minimized. The work in [11-13] focuses on minimizing the broadcast latency with multi-rate links. Broadcast latency is defined as the worst-case transmission time for delivering a packet from the root to all other nodes. As broadcast latency only measures packet-level end-to-end delay performance, it is not suitable for measuring large file/content transfer time [14]. For this reason, a widest spanning tree is proposed to minimize the content update time [14], where the width of a tree is defined as its bottleneck link bandwidth. In [15-17], real time broadcast call arrivals are considered. The design objective is to accept as many broadcast calls as possible.

While the spanning tree protocols above [9-17] are efficient in fulfilling their respective design objectives, they all assume that the number of interfaces at each node is pre-determined. Indeed, to maximize the network performance, the number of interfaces to be deployed at each node should be carefully designed [18]. The work in [18] focuses on enhancing unicast traffic in a WMN by equipping the congested nodes with extra interfaces. According to a given traffic profile and the routing decision, extra interfaces are added to the congested nodes to divide the loading into multiple collision domains. Different domains are then assigned with different channels. However, the number of interfaces (i.e. hardware cost) is not addressed. Also, since routing, interface assignment, and channel assignment are separately solved, the performance gain is also limited [16, 17].

Assume the total number of interfaces in the network is given, the problem of interface placement is to find the best way of placing interfaces to individual nodes such that the overall network performance is maximized. Take an example. Fig. 1(a) shows a 3-node network with pre-determined 2 interfaces per node. (Having identical number of interfaces per 
node is a widely adopted assumption [9-13].) Suppose we want to set up two connections, A-C and B-C. Fig. 1(a) shows a possible channel assignment, where A-C uses channel 1 and B-C uses channel 2. Although both nodes A and B each has a spare interface, they cannot use it because the two interfaces at $\mathrm{C}$ are already fully engaged. With interface placement, the total amount of 6 interfaces can be re-allocated as shown in Fig. 1(b). The resulting network performance is enhanced as three parallel transmissions are supported instead of two, and none of the interfaces are left idle.

In this paper, we focus on exploiting the flexibility brought by interface placement in constructing a widest spanning tree for efficient broadcast in multi-channel multi-interface WMNs. The problem of finding widest spanning tree is formulated as an Integer Linear Programming (ILP) problem, where joint optimization of interface placement, channel assignment, routing and scheduling is considered. By properly placing more interfaces to more "critical" nodes, the bandwidth of the spanning tree can be further increased.

The rest of the paper is organized as follows. The system model is introduced in Section II. In Section III, the ILP formulation for finding widest spanning tree is presented. Numerical results are given in Section IV. Finally, we conclude the paper in Section V.

\section{SYSTEM MODEL}

We consider a multi-channel multi-interface wireless mesh network (WMN), where the number of network interface cards (NICs) a node has can be different. A schedule-based MAC protocol is assumed, where the whole system works under a synchronized frame structure and conflict-free transmission is ensured by requiring links assigned with the same channel and within the mutual interference range to be active at different time slots. NICs can use multiple orthogonal channels for parallel transmissions. The channel switching latency is assumed to be small (compared to the session time) and thus ignored. The transmission range and interference range are assumed to be fixed and known in advance.

\section{A. Network Model}

We model the WMN by a connectivity graph $\boldsymbol{G}=(\boldsymbol{V}, \boldsymbol{E})$, where $\boldsymbol{V}$ and $\boldsymbol{E}$ represent the set of static mesh nodes and the set of unidirectional logical links, respectively. We denote $\boldsymbol{C}$ as the set of orthogonal channels available in the network, while the number of orthogonal channels $|\boldsymbol{C}|$ is determined by the wireless protocol. There are totally $T$ half-duplex NICs for the network, and the number of NICs allocated to node $u \in \boldsymbol{V}$ is denoted by $t_{u}$. For any two nodes $u, v \in \boldsymbol{V}$, there is a logical (directed) link $(u, v) \in \boldsymbol{E}$ if $u$ and $v$ are within the transmission range of each other. We assume symmetric connectivity, such that link $(u, v) \in \boldsymbol{E}$ if and only if $(v, u) \in \boldsymbol{E}$.

\section{B. Interference Model}

To capture the interference, the receiver conflict avoidance interference model [20] is adopted, which only requires the receiver to be clear for receiving. Let $\boldsymbol{I}_{\boldsymbol{v}}$ be the set of nodes within the interference range of node $v$. A data transmission from node $u$ to node $v$, i.e. data transmission on link $(u, v)$, is interfered by the data transmission on link $(a, b)$ if and only if $a \in \boldsymbol{I}_{\boldsymbol{v}}$.

\section{WIDEST SPANNING TREE FORMATION WITH INTERFACE PLACEMENT}

Given the connectivity graph $\boldsymbol{G}$, the orthogonal channel set $\boldsymbol{C}$, the total number of NICs in the network $T$, and the interference sets $\boldsymbol{I}_{\boldsymbol{u}}(\forall u \in \boldsymbol{V}$ ), our goal is to construct a widest spanning tree by joint optimization of interface placement, channel assignment, routing and scheduling. As the width of the tree is determined by the bottleneck link bandwidth, the problem is equivalent to maximizing the bottleneck link bandwidth of the spanning tree.

The following variables are used in our ILP formulation.

$t_{u}$ : integer variable; number of NICs to be placed at node $u$

$r_{u}$ : binary variable; equals to 1 if node $u$ is a root, 0 otherwise

$e_{u v}$ : binary variable; equals to 1 if link $(u, v)$ is on-tree, 0 otherwise

$s_{u}$ : voltage value $\left(0 \leq s_{u} \leq 1\right)$ assigned to node $u$ for loop prevention

$f_{u v}^{k}$ : time fraction for link $(u, v)$ to be active in carrying data using channel $k$

$n_{m v}^{k}$ : time fraction that node $m$ transmits on channel $k$ and interferes with the receiving at node $v$

$T$ : given total number of NICs in the network

$\alpha$ : a small constant ( $\alpha=0.0001$ is adopted in Section III)

$\beta$ : a large constant ( $\beta=10000$ is adopted in Section III)

Our ILP for finding the widest spanning tree is formulated below:

subject to:

$$
\operatorname{maximize}\{x\}
$$

$$
\begin{array}{cl}
\sum_{u \in \boldsymbol{V}} r_{u}=1 & \\
\sum_{(u, v) \in \boldsymbol{E}} e_{u v}=1-r_{v}, & \forall v \in \boldsymbol{V} \\
e_{u v}+e_{v u} \leq 1, & \forall(u, v) \in \boldsymbol{E} \\
s_{v}-s_{u} \geq \alpha e_{u v}-\left(1-e_{u v}\right), & \forall(u, v) \in \boldsymbol{E} \\
f_{u v}^{k} \leq e_{u v}, & \forall(u, v) \in \boldsymbol{E}, \forall k \in \boldsymbol{C} \\
\beta \sum_{k \in \boldsymbol{C}} f_{u v}^{k} \geq e_{u v}, & \forall(u, v) \in \boldsymbol{E} \\
f_{u v}^{k}-f_{u v}^{k} \leq 2-e_{u v}-e_{u w}, & \forall(u, v),(u, w) \in \boldsymbol{E}, \forall k \in \boldsymbol{C} \\
\sum_{u \in \boldsymbol{V}} t_{u} \leq T & \\
\sum_{k \in \boldsymbol{C}(u, v) \in \boldsymbol{E}} f_{u v}^{k}+\sum_{k \in \boldsymbol{C}} f_{v w}^{k} \leq t_{v}, & \forall v \in \boldsymbol{V}, \forall(v, w) \in \boldsymbol{E} \\
n_{m v}^{k} \geq f_{m n}^{k}, \quad \forall v \in \boldsymbol{V}, \forall m \in \boldsymbol{I}, \forall k \in \boldsymbol{C}, \forall(m, n) \in \boldsymbol{E} \\
f_{u v}^{k}+\sum_{m \in \boldsymbol{I}_{v}, m \neq u} n_{m v}^{k} \leq 1, & \forall(u, v) \in \boldsymbol{E}, \forall k \in \boldsymbol{C} \\
\beta\left(1-e_{u v}-e_{v u}\right)+\sum_{k \in \boldsymbol{C}}\left(f_{u v}^{k}+f_{v u}^{k}\right), & \forall(u, v),(v, u) \in \boldsymbol{E}
\end{array}
$$

The objective (1) is to maximize $x$, the bottleneck link bandwidth of the spanning tree as identified by the constraint (13). Note that $f_{u v}^{k} \in[0,1]$ is the portion of time (in a frame) 
for link $(u, v)$ to be active in carrying data using channel $k$. We have $f_{u v}^{k}=0$ if the link $(u, v)$ is not on the spanning tree. Therefore in constraint (13), off-tree links give a value of $\beta$ (a large constant) such that the bottleneck link bandwidth $x$ is determined by the on-tree links only. It should also be noted that links $(u, v)$ and $(v, u)$ are considered at the same time, as only one of them will be selected as an on-tree link.

Constraints (2)-(5) are topology constraints, which confine the final routing outcome to be a tree. Specifically, (2) states that there is only one root in the spanning tree, because the location of root is treated as a design parameter in our ILP. (If the root location is pre-determined, we can simply replace this constraint by setting the corresponding $r_{u}$ to 1 .) Constraint (3) specifies that exactly one ingress link can be assigned to each non-root node. Constraint (4) indicates that only one direction of the link should be used, as data flow is unidirectional and originated from the root. Constraint (5) ensures that no loop is formed in the routing path by forcing the sending node has a smaller voltage value than the receiving node. Note that the voltage at a node $s_{u} \in[0,1]$, if link $(u, v)$ is not on-tree, than no matter what the voltage values the two nodes have, their difference is always no less than -1.

Time fractions are assigned to the on-tree links according to the time fraction constraints (6)-(8). Constraints (6) states that if a link is not on-tree, no time fraction should be assigned to it. Otherwise, non-zero time fraction should be assigned, as specified by (7). With WBA, all nodes having the same parent node should be receiving at the same time while their parent is transmitting. Thus all egress links of a parent node should be assigned with the same time fraction. This WBA property is captured by constraint (8).

Constraints (9) and (10) are the NIC constraints. Constraint (9) states that the total number of NICs in the network should not be larger than $T$, the available number of NICs. Constraint (10) makes sure that no nodes in the network will be overloaded, i.e. the total amount of traffic to be carried by a node should not be larger than the total number NICs it has. Note that the first and second terms on the left hand side of (10) denote the total ingress and egress traffic at a node.

Constraints (11) and (12) are used to ensure that the assigned time fractions are schedulable. Recall that the receiver conflict avoidance interference model only requires the receiver to be clear for receiving. Constraint (11) finds $n_{m v}^{k}$ the total time fraction that a node $(m)$ is sending on channel $k$ (where the effect of WBA is considered). For every node $m$ within the interference range of a receiving node $v$, constraint (12) requires that the time fraction assigned to node $v$ for receiving on channel $k$ must be less than $1-\sum_{m \in I_{v}, m \neq u} n_{m v}^{k}$. This also provides the sufficient condition for the time fractions to be schedulable.

Theorem 1: If constraint (12) is not violated, then the assigned time fractions must be schedulable.

Proof: In a slotted system, the time fraction represents the portion of a frame. We can always find an integer $M$ such that all $f_{u v}^{k} M$ are integers, which also gives the number of time slots that link $(u, v)$ is active. Indeed, $M$ is the frame duration in slots. From constraint (12), we have, for any channel $k$

$$
f_{u v}^{k} M+\sum_{m \in I_{v}, m \neq u} n_{m v}^{k} M \leq M, \quad \forall(u, v) \in \boldsymbol{E} .
$$

In other words, the number of time slots assigned to link $(u, v)$ and the interfering transmissions is at most $M$, which is the number of time slots available within a frame duration. Thus the time fractions are schedulable.

\section{NUMERICAL RESULTS}

In this section, we evaluate the performance of different interface placement schemes in constructing the widest spanning tree. CPLEX11 [21] is used as our ILP solver. A $3 \times 4$ grid topology is used with grid length set to $200 \mathrm{~m}$. The transmission range and interference range are set to $250 \mathrm{~m}$ and $550 \mathrm{~m}$, respectively. There are 12 orthogonal channels available to the network, while the total number of NICs varies as specified in the simulation.

Fig. 2 shows the performance of different NIC placement schemes for constructing a widest spanning tree in a $3 \times 4$ grid network. In the figure, "NIC_place" stands for our proposed NIC placement; while "Fixed_equal" stands for the case that all nodes are equipped with the same number of NICs. For the two schemes, it is ensured that each node has at least one NIC. Note that we can easily pre-determine the number of NICs per node (for Fixed_equal) by setting $t_{u}$ to appropriate values.

From Fig. 2, it can be observed that the widest spanning tree with our NIC placement scheme achieves the highest bottleneck link bandwidth. When there are only 12 NICs in the network, the performance of the two NIC placement schemes are equal. The reason is due to the fact that every node has only $1 \mathrm{NIC}$, and no NIC placement is necessary. The result of widest spanning tree with 12 NICs is given by Fig. 3, where the root of the spanning tree is marked by a double-circle, the directions of the tree links are indicated by the arrows, and the time fractions assigned are labeled next to the corresponding tree links. As an example, the time fraction 0: 0.5 on the link from node 0 to node 3 means that this link is active on channel 0 for 0.5 time frame. Please note that our objective is to obtain a spanning tree with largest bottleneck link bandwidth. Thus the resulting tree is not necessary to be a shortest-path tree. From Fig. 3, we can see that every link can only be active for half of the time frame. This is because the single NIC on each node needs to spend half of the time for receiving and another half of the time for sending.

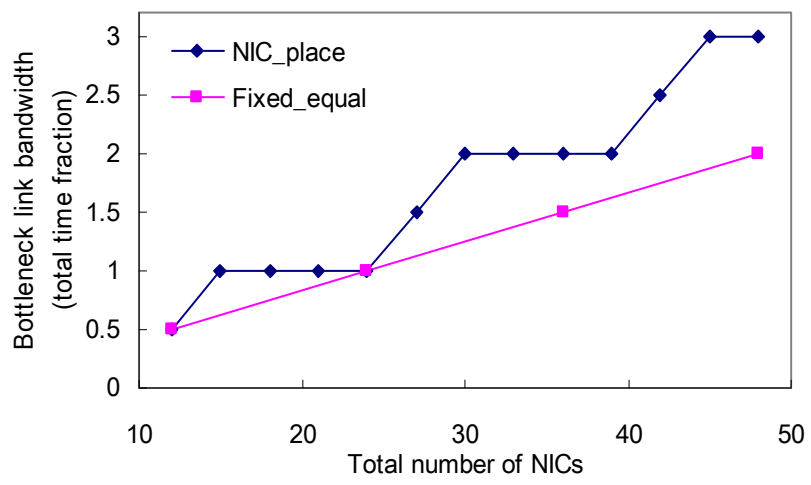

Fig. 2. Comparison of NIC placement schemes 
Widest Spanning Tree (with time fractions)

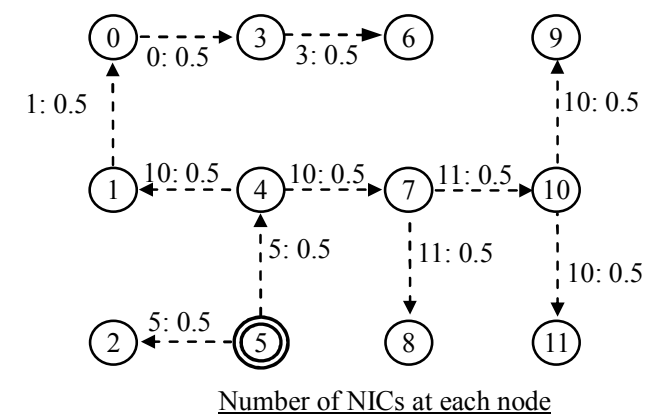

\begin{tabular}{|c|c|c|c|c|c|c|c|c|c|c|c|}
\hline$t_{0}$ & $t_{1}$ & $t_{2}$ & $t_{3}$ & $t_{4}$ & $t_{5}$ & $t_{6}$ & $t_{7}$ & $t_{8}$ & $t_{9}$ & $t_{10}$ & $t_{11}$ \\
\hline 1 & 1 & 1 & 1 & 1 & 1 & 1 & 1 & 1 & 1 & 1 & 1 \\
\hline
\end{tabular}

Fig. 3. Widest spanning tree with 12 NICs (no NIC placement is required)

Widest Spanning Tree (with time fractions)

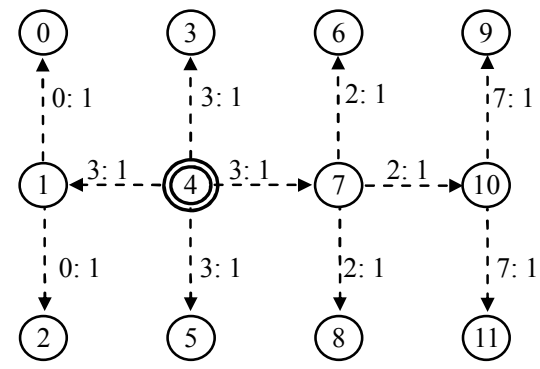

Number of NICs at each node

\begin{tabular}{|c|c|c|c|c|c|c|c|c|c|c|c|}
\hline$t_{0}$ & $t_{1}$ & $t_{2}$ & $t_{3}$ & $t_{4}$ & $t_{5}$ & $t_{6}$ & $t_{7}$ & $t_{8}$ & $t_{9}$ & $t_{10}$ & $t_{11}$ \\
\hline 1 & 2 & 1 & 1 & 1 & 1 & 1 & 2 & 1 & 1 & 2 & 1 \\
\hline
\end{tabular}

Fig. 4. Widest spanning tree with 15 NICs (proposed NIC placement)

On the other hand, if the network is provided with more NICs, NIC placement scheme plays an important role in enhancing the network performance. This performance gain can be observed by only adding 3 more NICs to the network. When there are 15 NICs, our NIC placement scheme can provide $100 \%$ performance improvement over the case of having 12 NICs. As shown in Fig. 4, the 3 extra NICs are placed in the critical part of the network, where the nodes along the center line (i.e. nodes 1,7 and 10) are equipped with 2 NICs such that they can have higher data forwarding capability. In this case, it can be observed that all tree links are active for the entire time frame, where the 2-NIC nodes can use 1 NIC for receiving and another NIC for sending.

For equal NIC placement to have the same performance, we must have 2 NICs/node, i.e. a total of 24 NICs provided to the network. This incurs more NIC overhead and hardware cost. Note that the extra NICs on the leaf-nodes remain idle and are wasted, since their upstream nodes can only transmit with a single NIC.

Another observation from Fig. 2 is that the performance of our proposed NIC placement scheme appears as a step-liked function. Hence, there is no further improvement on the bottleneck link bandwidth if we add less than 12 NICs to the network. Consider the network with 15 NICs in Fig. 4 again, where all NICs are fully utilized and the bottleneck link
Widest Spanning Tree (with time fractions)

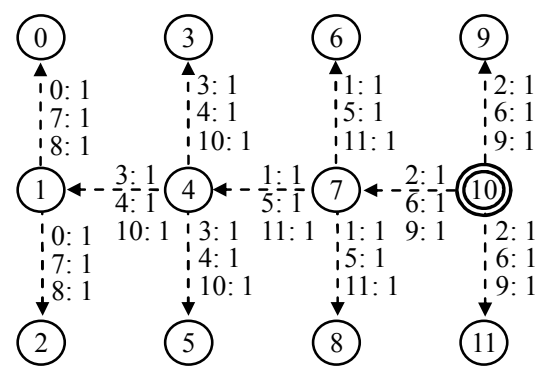

Number of NICs at each node

\begin{tabular}{|c|c|c|c|c|c|c|c|c|c|c|c|}
\hline$t_{0}$ & $t_{1}$ & $t_{2}$ & $t_{3}$ & $t_{4}$ & $t_{5}$ & $t_{6}$ & $t_{7}$ & $t_{8}$ & $t_{9}$ & $t_{10}$ & $t_{11}$ \\
\hline 3 & 6 & 3 & 3 & 6 & 3 & 3 & 6 & 3 & 3 & 3 & 3 \\
\hline
\end{tabular}

Fig. 5. Widest spanning tree with 45 NICs (proposed NIC placement)

bandwidth (time fraction) is 1 . If less than 12 extra NICs are added to the network, some nodes have extra NICs to transmit but the child nodes do not have extra NICs to receive, or vice versa. The resulting bottleneck link bandwidth remains unchanged, unless we add 12 or more NICs to the network. This also provides information of the suitable number of NICs such that no NIC is left idle.

In particular, as shown in Fig. 5, our proposed NIC placement scheme can fully utilize all the 12 channels with 45 NICs. For equal NIC placement scheme, it requires 72 NICs in order to have the same performance.

\section{CONCLUSION}

In this paper, we have investigated the construction of widest spanning tree in multi-channel multi-interface wireless mesh networks with interface placement. Unlike previous researches that assume the number of network interface cards (NICs) equipped on each node is a given parameter, we treat the number of NICs at each node as a design parameter, whereas the total number of NICs in the system is given. By properly allocating/placing more NICs to more "critical" nodes, the bandwidth of the spanning tree can be further maximized. To this end, a new Integer Linear Programming (ILP) is formulated for solving the widest spanning tree problem based on joint optimization of interface placement, channel assignment, routing and scheduling. We have evaluated the performance of our proposed interface placement scheme with the widely adopted equal placement scheme. Numerical results show that our proposed interface placement scheme provides a significant boost to the bandwidth of the widest spanning tree found.

\section{REFERENCES}

[1] H. Gossain, C. D. M. Cordeiro, and D. P. Agrawal, "Multicast: Wired to Wireless," IEEE Communications Magazine, vol. 40, issue 6, pp. 116-123, Jun 2002.

[2] Y.-Z. Huang, W. J. Liu, and K.-T. Feng, "Efficient Implementation of an Energy-conserving Multicast Routing Protocol for Wireless Multihop networks," Proc. of IEEE RWS, pp. 847-850, Jan 2008.

[3] J. Park and S. Sahni, "Maximum Lifetime Broadcasting in Wireless Networks," IEEE Transactions on Computers, vol. 54, no. 9, pp. 10811090, Sept 2005.

[4] A. S. Ahluwalia and E. H. Modiano, "On the Complexity and Distributed Construction of Energy-Efficient Broadcast Trees in Wireless Ad Hoc 
Networks," IEEE Transactions on Wireless Communications, vol 4, no. 5, pp. 2136-2147, Sept 2005.

[5] G. Deng and S. K. S. Gupta, "Maximizing Broadcast Tree Lifetime in Wireless Ad Hoc Networks," Proc. of IEEE Globecom'06, pp. 1-5, Nov 2006.

[6] Y. Li, T. Thai, F. Wang, and D.-Z. Du, "On the Construction of a Strongly Connected Broadcast Arborescence with Bounded Transmission Delay," IEEE Transactions on Mobile Computing, vol. 5, no. 10, pp. 1460-1470, Oct 2006.

[7] J. E. Wieselthier, G. D. Nguyen, and A. Ephremides, "Energy-Efficient Broadcast and Multicast Trees in Wireless Networks," Mobile Networks and Applications, 7(6): 481-492, 2002.

[8] P. Bahl, A. Adya, J. Padhye, and A. Wolman, "Reconsidering Wireless Systems with Multiple Radios," ACM Computer Communication Review, vol. 34, pp. 39-46, Oct 2004.

[9] J. He, J. Chen, and S. H. G. Chan, "Extending WLAN Coverage Using Infrastructureless Access Points," Proc. of IEEE HPSR'05, pp. 162-166, 2005.

[10] A. Raniwala and T. Chiueh, "Architecture and Algorithms for an IEEE 802.11-Based Multi-Channel Wireless Mesh Network," Proc. of IEEE Infocom 2005, vol. 3, 13-17, pp. 2223-2234, March 2005.

[11] J. Qadir, C. T. Chou, A. Misra, and J. G. Lim, "Localized MinimumLatency Broadcasting in Multi-radio Multi-rate Wireless Mesh Networks," Proc. of IEEE WOWMOM, pp. 1-12, June 2008.

[12] J. Qadir, A. Misra, and C. T. Chou, "Minimum Latency Broadcasting in Multi-Radio Multi-Channel Multi-Rate Wireless Meshes," Proc. of IEEE SECON 2006, pp. 80-89, 2006.

[13] M. Song, J. Wang, and Q. Hao, "Broadcasting Protocols for Multi-Radio Multi-Channel and Multi-Rate Mesh Networks," Proc. of IEEE ICC 2007, pp. 3604-3609, June 2007.

[14] H. S. Chiu, B. Wu, K. L. Yeung, and K.-S. Lui, "Widest Spanning Tree for Multi-Channel Multi-Interface Wireless Mesh Networks," Proc. of IEEE WCNC, pp. 2194-2199, 2008.

[15] C. T. Chou, B. H. Lui, and A. Misra, "Maximizing Broadcast and Multicast Trafic Load Through Link-Rate Diversity in Wireless Mesh Networks," Proc. of IEEE WOWMOM, 2007.

[16] H. S. Chiu, K. L. Yeung, and K.-S. Lui, "Maximizing Broadcast Load in Multi-Channel Multi-Interface Wireless Mesh Networks," Proc. of IEEE Globecom, 2008

[17] H. S. Chiu, K. L. Yeung, and K.-S. Lui, "'Bandwidth-Guaranteed Multicast in Multi-Channel Multi-Interface Wireless Mesh Networks," to appear in the Proc. of IEEE ICC, 2009.

[18] B. Aoun, R. Boutaba, and G. Kenward, "Analysis of Capacity Improvements in Multi-Radio Wireless Mesh Networks," Proc. of IEEE VTC, vol. 2, pp. 543-547, 2006.

[19] H. S. Chiu, K. L. Yeung, and K.-S. Lui, "J-CAR: An Efficient Joint Channel Assignment and Routing Protocol for IEEE 802.11-based Multi-Channel Multi-Interface Mobile Ad Hoc Networks," to appear in the IEEE Trans. of Wireless Communications, 2009.

[20] M. Kodialam and T. Nandagopal, "The Effect of Interference on the Capacity of Multi-hop Wireless Networks," IEEE Symposium on Information Theory, 2004.

[21] “ILOG CPLEX," http://www.ilog.com/products/cplex/. 